\title{
Mucormycosis in diabetes mellitus
}

Prashant Khatiwada, ${ }^{* a}$ Aditi Giri, ${ }^{a}$ Prasana Khatiwoda, ${ }^{b}$

${ }^{a}$ Kathmandu University School of Medical Sciences, Dhulikhel, Nepal

${ }^{b}$ Richa Bajimaya Memorial Foundation, Kathmandu, Nepal

\section{Date of acceptance}

April 13th, 2012

\section{DOI Name}

10.3126/jaim.v1i2.6531

\section{Keywords}

fungal infection, immunodeficiency,

mucormycosis

\section{Citation}

Khatiwada P, Giri A, Khatiwoda P. Mucormycosis in Diabetes Mellitus. Journal of Advances in Internal Medicine 2012;01(2):73-5.

\begin{abstract}
Mucormycosis is an opportunistic fulminant fungal infection, which mainly infects immunocompromised patients. Infection with mucormycosis can cause devastating rhino-orbital-cerebral and pulmonary infection with an extremely poor prognosis. The infection begins in the nose and paranasal sinuses due to inhalation of fungal spores. We report a case of mucormycosis in an uncontrolled diabetic patient to emphasize early diagnosis of this potentially fatal fungal infection. Rhino-orbitalcerebral mucormycosis is the most common manifestation in patients with diabetes mellitus. We also review the current concepts in management of mucormycosis. Mucormycosis remains difficult to treat and requires a multifaceted approach involving elimination of predisposing factors, surgical debridement, and anti-fungal therapy. Lipid formulations of Amphotericin B are the treatments of choice.
\end{abstract}

\section{INTRODUCTION}

Mucormycosis is rare fungal infection caused by several fungi, including Mucor species, Rhizopus species and Absidia species. Recognized as one of the most rapidly progressive lethal form of fungal infection in humans with a high mortality of 25-62 percent. ${ }^{1-6}$ It occurs in severely ill patients with hallmark of the disease being vascular invasion with marked hemorrhagic necrosis. Among the conditions predisposing to Mucormycosis are diabetes mellitus, malnutrition, hematological malignancies, neutropenia, burns, surgical procedures, occlusive dressings, antibiotics, long term steroid therapy and immunosuppressive therapy., ${ }^{4,-17}$ The usual presentation is acute infection, first localized to rhino-cerebral area, gradually spread to surrounding areas, or even dissemination and affecting distant organs. In the case presented here the infection follows acute course, and somewhat indolent form which eventually caused maxillary necrosis.

\section{CASE REPORT}

A 24 years old female presented to Kathmandu University Hospital (Dhulikhel, Nepal) in July 2010 with a history of facial swelling, progressive loss of vision in the left eye associated with a week long history of headache and facial pain. There was swelling for 15 days, progressive in nature, left facial involvement, with pus discharge from left nose and ear. Facial pain localized in the left upper facial region, moderate in nature, aggravated on bending the head and chewing food. The patient also complained of nasal congestion, earache and fever with chills and rigors.

She was a diagnosed case of poorly controlled diabetes mellitus for four years, on irregular treatment with oral hypoglycemic drugs and insulin therapy. She has a history of surgical removal of right eye, for loss of vision and infection on right eye due to opportunistic diabetic infection. There is also history of admission due to diabetic ketoacidosis on three previous occasions. Her mother, was also a sufferer of Diabetes Mellitus Type two, and died due to its complications at the age of 52 years.

On general examination, patient had high grade fever of 103 degree Fahrenheit, ill looking with generalized body swelling, bilateral pedal pitting edema, with marked swelling in the left periorbital area, upper lip, and zygomaticomaxillary complex region. There were pus points in left upper fa- cial area with multiple pleomorphic black patches; pus discharge from left ear and nasal openings. Oral examination revealed perforated hard palate with ulceration and grayish white and black patches. A detailed ophthalmological examination confirmed left eye orbital cellulitis, with proptosis and peri orbital swelling; presence of chemosis and corneal edema, fixed and dilated pupil with frozen globe. Systemic examination of cardiovascular, pulmonary and gastrointestinal system was within normal limits.

Biochemical investigations revealed elevated blood sugar level, random blood sugar of $401 \mathrm{mg} / \mathrm{dl}$. White blood cell count of $2700 / \mathrm{mm}^{3}$ with $90 \%$ neutrophil and $10 \%$ lymphocytes, hemoglobin of $5.3 \mathrm{gm} / \mathrm{dl}$, Urea $41 \mathrm{mg} /$ $\mathrm{dL}$ and Creatinine $1 \mathrm{mg} / \mathrm{dL}$. Electrolytes were at level of $130 \mathrm{mEq} / \mathrm{dL}$ for Sodium and $3.9 \mathrm{mEq} / \mathrm{dL}$ for Potassium. Urine ketone bodies were present significantly and the Arterial Blood Gas (ABG) analysis indicated a state of severe uncompensated metabolic acidosis. Urine culture revealed Klebsiella oxytica sensitive to Gentamycin. Swab culture from palate revealed multiple organisms- Kleibsiella pneumonea, Escherichia coli, and Methicillin resistant Staphylococcus aureus sensitive to Gentamicin. Swab culture from nose contained Escherichia coli.

Palate biopsy confirmed the presence of necrotic tissue mixed with fibrous tissue and non-septate fungal hyphae. Hyphae showed irregular width with right angle branching. A diagnosis of mucormycosis was made on the basis of the morphology of the fungal hyphae. Computer tomography scan of paranasal sinuses revealed large soft tissue lesion in anterior aspect of hard palate associated with bony destruction, diffuse soft tissue opacification involving left nasal cavity. Mucosal thickening involved bilateral maxillary sinuses with widening of left ostium and infundibulum, bilateral ethmoidal sinuses (left more than right) and both halves of sphenoid sinuses. Also seen in CT Scan were right phthisis bulbus, mild left sided proptosis with bulky lacrimal gland, and no obvious intracranial le-

\footnotetext{
* Corresponding author

Prashant Khatiwada

Kathmandu University School of Medical Sciences, Dhulikhel, Nepal

Emailaddress - prashant@richafoundation.org.np
} 
sion/extension. Management of diabetic ketoacidosis was initiated within the first six hours of presentation, with intra venous fluid therapy, Insulin and potassium replacement. Parental Amphotericin B was initiated as anti fungal therapy with infusion in 5 percent dextrose solution at $6 \mathrm{drops} / \mathrm{min}$ (slow infusion) via central venous line. Care was taken to avoid exposure of Amphotericin to sunlight and the intravenous drip set was adequately covered. Serum potassium levels were monitored to look out for hypokalemia. Blood sugars were maintained with subcutaneous insulin administration and oral hypoglycemic agents. Antibiotic therapy was initiated with combination of Amoxicillin and Clavulinic acid, Metronidazole and Gentamicin after culture reports. Regular nasal endoscopy and removal of crusts performed along with saline nasal douching. Patient was treated for next twelve days, stabilizing her condition, before she was referred to specialist health care center for left eye and paranasal sinus surgery.

\section{DISCUSSION}

Mucormycosis is manifested by a variety of different syndromes in humans, predominantly in immunocompromised patients and those with diabetes mellitus. ${ }^{2}$ Devastating rhino-orbital-cerebral and pulmonary infections are the most common conditions caused by these fungi. These belong to the order Mucorales. The genera most commonly found in human infections are Rhizopus, Mucor, and Rhizomucor. ${ }^{3,4}$ Found ubiquitous in nature, humans have plenty of exposure to these fungi during day-today activities with effective and intact human immune system against it. Almost all patients with invasive mucormycosis have some underlying disease that both predisposes to the infection and influences the clinical presentation.

Rhizopus organisms have an enzyme, ketone reductase, which allows them to thrive in high glucose, acidic conditions. Serum from healthy individuals inhibits growth of Rhizopus, whereas serum from individuals in diabetic ketoacidosis stimulates growth. ${ }^{5}$ The agents of mucormycosis are angioinvasive and infarction of infected tissues is a hallmark of invasive disease. ${ }^{18,19}$ A review of 929 cases of mucormycosis that were reported since 1940 noted that diabetes was the most common risk factor, found in 36 percent of cases, followed by hematologic malignancies (17 percent), and solid organ or hematopoietic stem cell transplantation (12 percent). ${ }^{4}$ In some patients, mucormycosis was the diabetes-defining illness. ${ }^{20}$

Mucormycosis is characterized by infarction and necrosis of host tissues that results from invasion of the vasculature by hyphae. Rhino-orbitalcerebral infection being most common clinical presentation is presumed to start with inhalation of spores into the paranasal sinuses of a susceptible host. Hyperglycemia, usually with an associated metabolic acidosis, is the most common underlying condition. ${ }^{4}$ The infection presents as acute sinusitis with fever, nasal congestion, purulent nasal discharge, headache, and sinus pain. ${ }^{20,21}$ All of the sinuses become involved and spread to contiguous structures, such as the palate, orbit and brain, usually progresses rapidly. The hallmarks of spread beyond the sinuses are tissue necrosis of the palate resulting in palatal eschars, destruction of the turbinates, perinasal swelling, and erythema and cyanosis of the facial skin overlying the involved sinuses. ${ }^{20} \mathrm{~A}$ black eschar, which results from necrosis of tissues after vascular invasion by the fungus, may be visible in the nasal mucosa or the palate. Apart from rhino-orbital-cerebral infection other sites of infection also include - pulmonary system, gastrointestinal tract, skin, kidneys and isolated CNS infection. ${ }^{1,2,4,5}$ Disseminated mucormycosis is rare, and occurs most commonly in severely immunocompromised patients, burn patients, premature infants, and individuals who have

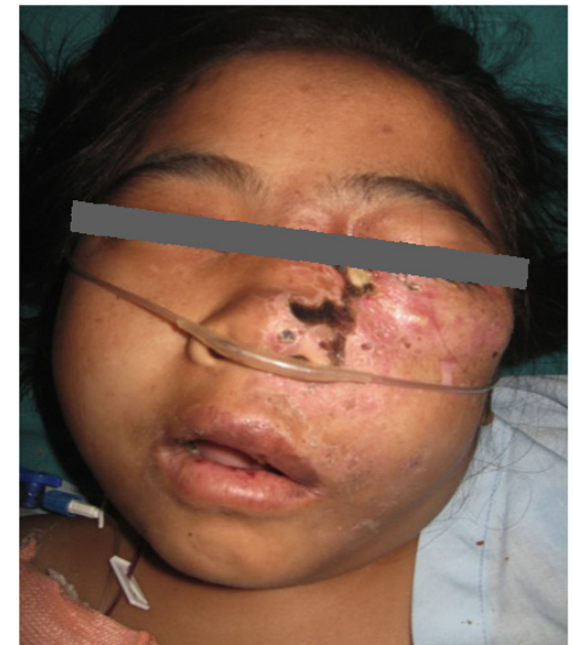

Figure 1. Presentation of the patient

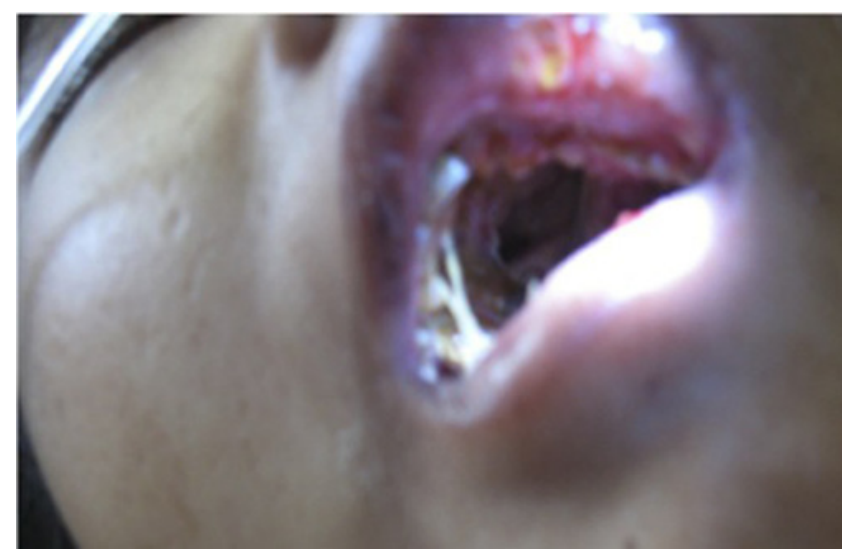

Figure 2. Oral examination reveals extensive infection in hard palate

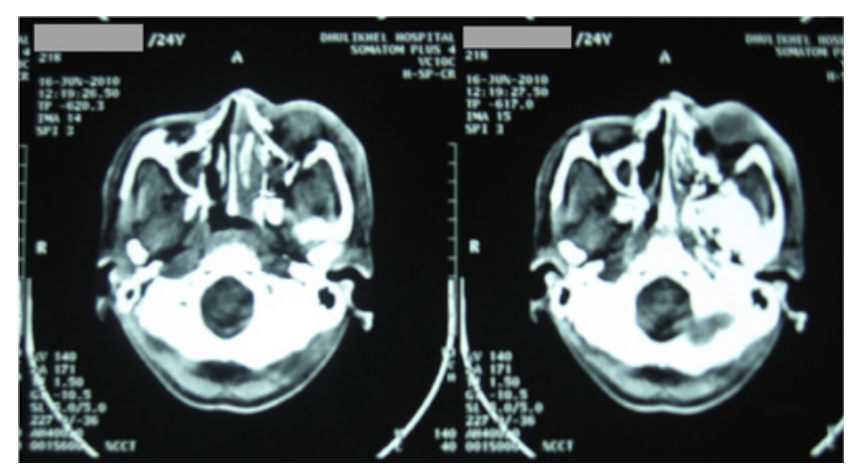

Figure 3. CT Scan showing extensive paranasal sinus involvement with extension up to optic cavity

received deferoxamine. ${ }^{1,4}$ Deferoxamine, which chelates both iron and aluminum, increases the risk of mucormycosis by enhancing growth and pathogenicity. ${ }^{8,18,22}$ The deferoxamine-iron chelate, called feroxamine, is a siderophore for the species Rhizopus, increasing iron uptake by the fungus, which stimulates fungal growth and leads to tissue invasion. ${ }^{7,8,19}$

The diagnosis of mucormycosis relies upon the identification of organisms in tissue by histopathology with culture confirmation. However, culture often yields no growth, and histopathological identification of 
an agent of mucormycosis may provide the only evidence of infection. A clinician must think of this entity in the appropriate clinical setting and pursue invasive testing in order to establish a diagnosis as early as possible. Treatment involves a combination of surgical debridement of involved tissues and antifungal therapy. Elimination of predisposing factors for infection such as hyperglycemia, metabolic acidosis, deferoxamine administration, immunosuppressive drugs, and neutropenia is also critical. Intravenous Amphotericin B (lipid formulation) is the drug of choice. ${ }^{11,12,21,22}$ Aggressive surgical debridement of involved tissues should be undertaken as soon as the diagnosis of rhino-orbitalcerebral mucormycosis is suspected. ${ }^{17,20}$ The debridement to remove all necrotic tissue will often be disfiguring, requiring removal of the palate, nasal cartilages, and the orbit. Intravenous Amphotericin B (lipid formulation) is the drug of choice. Lipid formulation of Amphotericin $B$ is used in order to deliver a high dose with less nephrotoxicity. The usual starting dose is $5 \mathrm{mg} / \mathrm{kg}$ daily of liposomal Amphotericin B or Amphotericin B lipid complex, with increase in dose as high as $10 \mathrm{mg} / \mathrm{kg}$ daily in an attempt to control this infection. ${ }^{22-25}$

Despite early diagnosis and aggressive combined surgical and medical therapy, the prognosis for recovery from mucormycosis is poor. Overall mortality from rhino-orbital-cerebral mucormycosis ranges from 25 to 62 percent, with the best prognosis in patients with infection confined to the sinuses. ${ }^{4,17,21,22}$ The prognosis is especially poor for patients with brain, cavernous sinus or carotid involvement, although some patients with these complications have been cured of the infection. The outcome in patients with pulmonary mucormycosis is worse than for patients with rhino-orbital-cerebral involvement, with mortality rates as high as 87 percent. $^{20}$

\section{CONCLUSION}

This case study highlights a rare case of mucormycosis of the paranasal sinuses in a relatively young diabetic patient. Depending on the underlying condition of immune compromised non hematological patients, mucormycosis can manifest in various clinical forms: mostly as rhino-orbital or rhino-cerebral in diabetes patients. Knowledge of the differential patterns of clinical presentation can raise the suspicion index for these rare but highly lethal infections. This is very important since prompt and appropriate management can reduce mortality and morbidity considerably. In the absence of large/tertiary health centers, treatment of this infection should be individualized. A multi-disciplinary team approach to identify early complications and sequelae is strongly recommended.

\section{REFERENCES}

1. Spellberg B, Walsh TJ, Kontoyiannis DP, et al. Recent advances in the management of mucormycosis: from bench to bedside. Clin Infect Dis 2009;48:1743-51.

2. Kauffman CA, Malani AN. Zygomycosis: an emerging fungal infection with new options for management. Curr Infect Dis Rep 2007; 9:435-40.

3. Hibbett DS, Binder M, Bischoff JF, et al. A higher-level phylogenetic classification of the Fungi. Mycol Res 2007; 111:509-47.

4. Roden MM, Zaoutis TE, Buchanan WL, et al. Epidemiology and outcome of zygomycosis: a review of 929 reported cases. Clin Infect Dis 2005;41:634-53.

5. Gale GR, Welch A. Studies of opportunistic fungi. Am J Med 1961; 45:604-12.

6. Bitar D, Van Cauteren D, Lanternier F, et al. Increasing incidence of zygomycosis (mucormycosis), France, 1997-2006. Emerg Infect Dis 2009;15:1395-401.

7. Boelaert JR, Fenves AZ, Coburn JW. Deferoxamine therapy and mucormycosis in dialysis patients: report of an international registry. Am J Kidney Dis 1991;18:660-7.

8. Boelaert JR, M de Locht, Van Cutsem J, et al. Mucormycosis during deferoxamine therapy is a siderophore-mediated infection. In vitro and in vivo animal studies. J Clin Invest 1993; 91:197986.

9. Koklu E, Akcakus M, Torun YA, et. al. Primary Gangrenous Cutaneous Mucormycosis of the Scalp in a Child. Pediatric Emergency Care 2008;24:102-4.

10. Chan LL, Singh S, Jones D, et al. Imaging of Mucormycosis Skull Base Osteomyelitis. Am J Neuroradiol 2000;21:828-31.

11. Ibrahim AS, Edwards JE Jr, Fu Y, et al. Deferiprone iron chelation as a novel therapy for experimental mucormycosis. J Antimicrob Chemother 2006;58:1070-3.

12. Ibrahim AS, Gebermariam T, Fu Y, et al. The iron chelator deferasirox protects mice from mucormycosis through iron starvation. J Clin Invest 2007;117:2649-57.
13. McNulty JS. Rhinocerebral mucormycosis: predisposing factors. Laryngoscope 1982;92:1140-3.

14. Chakrabarti A, Das A, Mandal J, et al. The rising trend of invasive zygomycosis in patients with uncontrolled diabetes mellitus. Med Mycol 2006;44:335-42.

15. Elinav H, Zimhony O, Cohen MJ, et al. Rhinocerebral mucormycosis in patients without predisposing medical conditions: a review of the literature. Clin Microbiol Infect 2009;15:693-7.

16. Rajagopalan S. Serious infections in elderly patients with diabetes mellitus. Clin Infect Dis 2005;40:990-6.

17. Yohai RA, Bullock JD, Aziz AA, et al. Survival factors in rhinoorbital-cerebral mucormycosis. Surv Ophthalmol 1994;39:3-22.

18. Ferguson BJ. Mucormycosis of the nose and paranasal sinuses. Otolaryngol Clin North Am 2000;33:349-65. mycosis): emerging clinical importance and new treatments. Curr Opin Infect Dis 2004;17:517-25.

20. Harril WC, Stewart MG, Lee AG, et al. Chronic rhinocerebral mucormycosis. Laryngoscope 1996;106:1292-7.

21. Walsh TJ, Kontoyiannis DP. Editorial commentary: what is the role of combination therapy in management of zygomycosis? Clin Infect Dis 2008;47:372-4.

22. Strasser MD, Kennedy RJ, Adam RD. Rhinocerebral mucormycosis. Therapy with amphotericin B lipid complex. Arch Intern Med 1996;156:337-9.

23. Boelaert JR, Van Cutsem J, de Locht M, et al. Deferoxamine augments growth and pathogenicity of Rhizopus, while hydroxypyridinone chelators have no effect. Kidney Int 1994;45:667-71.

24. de Locht M, Boelaert JR, Schneider YJ. Iron uptake from ferrioxamine and from ferrirhizoferrin by germinating spores of Rhizopus microsporus. Biochem Pharmacol 1994;47:1843-50.

25. Kontoyiannis DP. Decrease in the number of reported cases of zygomycosis among patients with diabetes mellitus: a hypothesis. Clin Infect Dis 2007;44:1089-90.
19. Greenberg RN, Scott LJ, Vaughn HH, et al. Zygomycosis (mucor- 